\title{
Evidence in Surgery—Levels and Significance
}

\section{Sandeep Kumar ${ }^{1}$}

Published online: 6 August 2019

(C) Association of Surgeons of India 2019

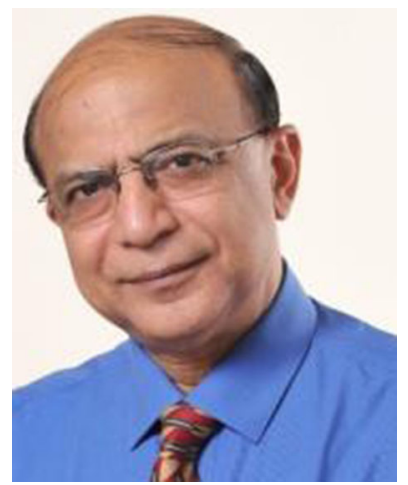

The past two decades of modern medical practice has witnessed increasing use of the term evidence-based medicine (EBM). This was inadvertently defined by David Sackett et al. in 1996 as 'A systematic approach to clinical problem solving by the integration of best research evidence with clinical expertise and patient values' [1]. Yet another definition emerged from Dr. Gordon Guyatt's statement: 'The conscientious, explicit and judicious use of current best evidence in making decisions about the care of the individual patient' [2]. Both these definitions have the same implications. It means consolidated individual clinical experience with the best available external clinical evidence from systematic research in a given situation. Therefore, the three important components of EBM are best research evidence, clinical expertise and patients' values and preferences. This fundamental principle of evidence-based practice is imperative for today's surgeon. Application of 'best evidence' if used judiciously results in improved standards of surgical care. Surgical training and teaching should also be evidence based, for example, highfidelity simulation laboratories, for laparoscopic, robotic and

Sandeep Kumar

profsandeepsurgeon@gmail.com

1 Saraswati Medical College Unnao, Unnao, Uttar Pradesh, India micro-surgical training has become important. The question arises what is research? A good answer is 'focussed systematic enquiry generating new knowledge to improve health, treatment outcomes and patient satisfaction'.

Fears were raised by the proponents of EBM that it may be pronounced as old wine in a new glass or even a dangerous innovation perpetrated by arrogant clinicians and cost cutter hospital managers, and it will suppress clinical freedom. This view about EBM has continued even today [3]. More so recently, voices have been raised by some eminent medical personalities to admonish EBM and have reported somewhat doubtful trustworthiness of EBM [3, 4]. High-quality evidence requires long-term studies with follow-up and randomised clinical trials. These require organised efforts and large inputs of manpower and money. It is now being said that EBM has been hijacked by rich and powerful pharmaceuticals [4]. Randomised trials said to be ultimate to produce evidence of efficacy have also been questioned [5, 6]. However, the tenets of EBM have undoubtedly made physicians and practitioners wiser and alert to what they prescribe. There are also certain limitations of the evidence-based practice through external research. It has pervaded the surgical fraternity to a lesser extent as compared with internal medicine practice.

Evidence by itself does not make the decisions but it can help and support the patient care process. The 'three components' of modern clinical practice are clinical expertise along with available resources at the time of delivery of healthcare, best research evidence and patient values and preferences. This in theory should achieve the best safety for the care provider and achieve appropriate outcome for the patients in the given circumstances. In this new paradigm, a modern surgeon should be introspective and honestly understand his strengths and weaknesses of clinical expertise and resources available to him. The doctors should also acquire adroitness in logical literature searching and the application of formal rules of evidence in evaluating the clinical literature. Traditionally however, the treating surgeons are known to respect the preferences and values of patients and their attendants. The choice of the patient is of paramount importance. For example, open or laparoscopic 
surgery or whether to go for robotic surgery or not or to have a breast reconstruction often is decided by patients.

It is no longer considered adequate to treat a patient purely on clinical experience alone, without a clear affirmation of evidence based on research. An examination of 'evidencebased practice' shows that it has usually been shifted through the opinions of experts and journal editors, and 'opinion-based medicine' as a term was suggested but generally not accepted. Yet another term has been advocated by Ayush researchers called 'comparative effectiveness research (CER) [7]. The Ayurvedic medicines are there in a big way not only in Indian markets but also abroad. There are only few alternative medicines - mostly from China that have been taken through the rigour of RCTs [8]. Those Ayurvedic medicines where standardised preparation, distillates and lyophilised salts have been prepared are readily adopted in modern medicine. They undergo RCTs by modern physicians. CER [7] is just a term coined where there is serious lacuna of a 3rd party audit, strict inclusion and exclusion criteria, data safety monitoring, statistical evaluation of the size effect and availability of the data for subsequent visits. Ayush medicines need to be scrutinised by RCTs and standardised using GMP (good manufacturing practices). Another surgical treatment first described in
Ayurveda is the use of kshar sootra - an alkaline seton which has cutting property for the treatment of chronic anal fistulae. The kshar sootra has been tested by several laboratory studies, observational studies and a few trials. This led to a wide spread adoption of kshar sootra in modern surgical practice also. Several other extended uses of kshar sootra have come through individual practitioners in other indications through observational studies.

\section{Levels and Hierarchy of Evidences}

The following table shows American Society of Clinical Oncology Guidelines for the levels of evidence and grades of recommendation to undertake interventions based on these levels. This method of graded recommendations may look somewhat pedantic and at times may preclude innovations. Such a hierarchical mandate as given below can be threatening to some. An Oxford centre for EBM [9] proposed an evidence pyramid given below.

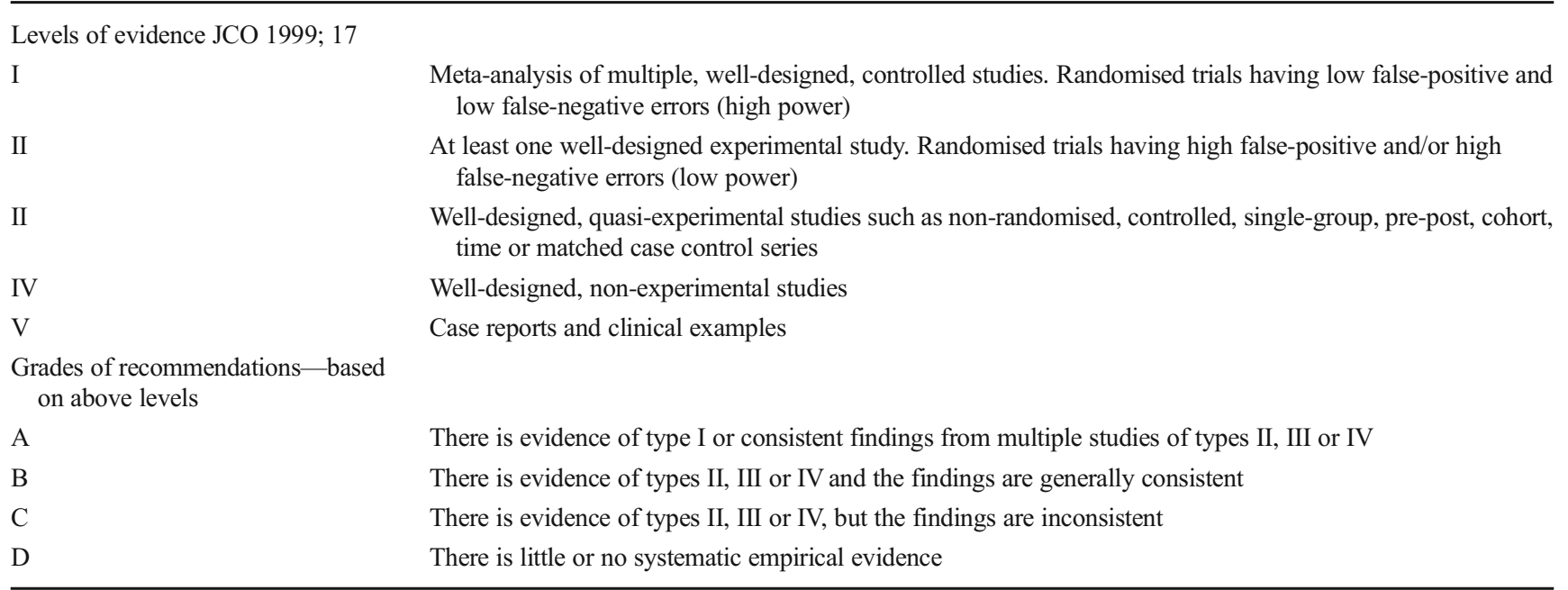

\section{Why Do We Need Evidence-Based Practice?}

The advantages of evidence-based practice include desirable patient outcomes, increased patient welfare and improved quality of life. In the past, patients simply received healthcare. Today, patients are healthcare consumers. They demand improved treatments and increased safety. Evidence-based practice endeavours to standardise practices, which can deliver more foreseeable outcomes. The meticulous approach of this type of practice and research can result in treatments with improved prospects of success. The basis of this practice lies in research that provides well-grounded information about treatments. Analysing this information in light of a patient's situation can determine the likelihood of a positive result. Evidence-based practices are putatively patient-centric philosophy addressing the needs of the patient with the goal of more 
efficient treatment. Other benefits of evidence-based practice include decreased healthcare cost by using principles of health economics. A lot of clinical trials have in-built costeffectiveness, cost-benefit or cost-utility studies. Use of highend medical equipment and technology at times may simply escalate cost without improving the outcome. For example, use of laser to extirpate leaking veins of legs in patients of venous insufficiency costs much more as compared with surgical venous stripping and sclerosing solution injections that achieve equal or better results. A large number of high-profile trials that show marginal-to-moderate benefit or equivocal outcomes fail to convince the doctors to change practice. Some examples are medical versus surgical treatments for particular level of coronary artery disease, joint replacement surgery, birthing practices and even routine vaccination regimens. A common saying is 'you get the treatment according to the door you enter'. A medical conservative cardiologist will control his patients on medical treatment including counselling, strict dietary and activity-related advises. An interventionist cardiologist is most likely to do an angioplasty and stent, whereas a cardiac surgeon may straight away suggest bypass surgery. They continue to do what they are most comfortable with. Further justification is given for their decision as they are able to find one or the other study in the literature to support their point of view. This allowed doctors and surgeons, in particular, a reasonable freedom to do what they want to do and yet continue to embrace EBM.

Evidence-based practice has today spread beyond medicine and surgery into disciplines like nursing, psychology and education. The early-day practices of performing tonsillectomies nearly for everybody and neck irradiation for sore throat leading to thyroid cancer were surrendered after watchful result appraisal. These were perhaps the earlier use of EBM when the term was not coined [10].

\section{How Do We Practice EBM?}

There is a 5-step approach to practice EBM. These include the clinical assessment by history taking, physical examination and diagnostic tests and convert this information into an answerable clinical question. In the 2 nd step, we search for the best evidence in literature to answer this. We will then see if this evidence is really applicable to the scenario of the patients that we are treating. At the 4th step, we will still be masters of our clinical decision and may perform better if we integrate the relevant, valid and applicable research evidence into clinical practice. The final step is to continue to strive to work in this itinerant loop and improve the patient outcome with each application. A lot of clinical events and their outcomes are complex in nature and difficult to evaluate. Despite the sincere use of the above 5 steps, the flip side is that there is no method to know when our observations about occurrence of complex events in nature are complete. Our knowledge is definable, but our ignorance is indefinite. In medicine, we can never be unquestionable about the consequences of interventions and we can only narrow down the area of uncertainty.

\section{Practicing EBM-the 5-Step Approach [11]}

The most important is to formulate a research question that is highly specific to the situation. In an acronym PICO, P stands for patient population or group for which evidence is required, I stands for intervention/operation/ treatment - the effect of which is to be evaluated, $\mathrm{C}$ stands for comparison between the two interventions/operations/ treatment or non-operative versus operative treatment or placebo versus treatment, and $\mathrm{O}$ stands for outcome or end points and effects of the treatment or surgery [12].

\section{Can Surgeons Practice EBM?}

There is a considerable gap between what we know from research and what is done in clinical practice. This gap is more pronounced in surgical fraternity compared with other departments. One cause of the gap in evidence-based practice by surgeons is information overload; for example, approximately 8000 references including around 350 randomised trials are added to MEDLINE each week. But only a small bit of this is research that is well founded and relevant to change practice. So keeping all surgeons and practitioners up-to-date with new developments and data is difficult. One arm of EBM has been to synthesise and summarise this outpouring of research and be able to access evidence wherever and whenever it is needed. To achieve this access to sources, evidence requires the resources such as MEDLINE and the Cochrane Library [13]. It also requires acquiring the skills in finding, appraising and applying evidence that few healthcare workers currently have. The EBM movement has focused on developing both the skills and tools to better connect research and clinical practice, with some but not universal successes. A yet another gap is that a lot of research that is truly worthy of adopting and adapting remain unutilised or underutilised. A new effort called 'practice change' or 'translation research' is in current use to forge utilisation of best research findings. It may be noteworthy that a huge amount of well-acknowledged research findings that are in practice at some places and are well known to achieve better outcomes are not universally practiced. Majority of good and sincere surgeons and physicians are often inundated with a large number of patients and have little or no available time. Therefore, we find many more questions are not answered for want of time by role model surgeons. It is erroneous to state that the hard headedness of surgeons leads them to denounce EBM. Certain strategies like capturing or saving, scheduling and selecting, by employing clinical epidemiologists and hospital managers, making them 
write standard operating practices and treatment guidelines have been used to overcome these $[14,15]$.

Are surgeons are known to produce weakest evidence in literature if one goes by the levels of evidence pyramid shown above? [15] Such a statement has been made however, no evidence to this effect has been cited and we do not know of any research which shows that surgeons produce the weakest evidence and do not indulge in experimental and epidemiological studies. The following are some of the reasons to show why EBM is not a perfect fit in surgery:

- Historically, many operations develop over several years with minor evolutions in the surgical steps or approach; for example, nothing has changed as regards surgery for gall bladder stone. The gall bladder is removed after ligating the cystic artery and duct with careful dissection from the liver bed. You do this as a hilum first or fundus first method. In the past four decades, the approach changed from paramedian incision to midline incision to sub-costal Kocher's incision to mini-lap incision to laparoscopic or robotic cholecystectomy. Within the laparoscopic cholecystectomy, one can debate from how many ports you make and how to perform a cholecystectomy. From the EBM point of view, the primary objective of safe removal of the gall bladder is achieved in all the above approaches and whatever evidence showing superiority of one over other is based on minor secondary outcomes.

- Also, some surgeons acquire skills and perfection to perform a particular surgery that can excel the results of a poorly performed more evolved surgical procedure. This is called efficacy of a procedure by an expert who has mastered it. Many surgeons even today would perform the age-old Bassini's repair for inguinal hernia with excellent results [16]. Whereas surgeons trained and given to using a mesh hernioplasty may produce equivalent research because of their training and experience in doing that particular procedure, however they will not be able to achieve the same results with Bassini's type repair. A dilettante use of the same operation will label that procedure as inferior or a complete failure. It thus becomes difficult to do epidemiologically robust comparison of surgical procedures.

- The learning curve is different for different surgeons and at some centres, the number of cases can be accrued in a shorter period of time. This again makes comparison based on years of experience incorrect. It is also very hard to judge the surgical competencies.

- The patients also do not like their operations to be selected by randomisation.

- The operating conditions for the same surgeon may vary in a private vis-a-vis public sector hospitals.
- The operating skills during emergency or elective surgery for the same condition are somewhat different and require a different set up of back up for surgery.

- Surgical RCTs mostly will not have external funding as there is no vested interest of a pharmaceutical company to fund these. Therefore, fewer RCTs in surgery are performed. Use of mesh versus no-mesh hernia repairs has been a subject of several RCTs as these may have been done on the behest of surgical hernia mesh making companies.

- That surgeons are arrogant and they abhor subjecting their doings to trial and someone else to evaluate their doings, are poor students of biostatistics and would not agree to be audited are some of the charges that have been used in literature from time to time. However, the authors here do not hold such a view. Surgical fraternity is equally or more open to audit. In fact, the surgical outcomes are highly visible outcomes and these are externally audited all the time. Poor results will automatically boomerang on a surgeon's reputation. Surgeons quickly adapt and adopt. The fact that India did not lack behind in embracing laparoscopic and robotic surgery is the testimony to the highly receptive surgical fraternity.

- It will be hard for any surgeon to state before or after a surgery that a procedure was performed with a particular grade of recommendation- $\mathrm{A}, \mathrm{B}, \mathrm{C}$ or $\mathrm{D}$ as shown above.

\section{Limitation of Evidence-Based Practice?}

EBM has the limitations in surgery as discussed above. Surgeons also prescribe treatment for common comorbid illnesses like mild-to-moderate diabetes, hypertension, depression and rheumatism. How can one expect that surgeons keep abreast of only with most recent surgical advances and evidences in the management of surgical conditions but also for all the concomitant illnesses that are accompaniments of surgical conditions? Surgeons frequently prescribe diagnostic tests in parallel-serial-parallel manner. To get to a final diagnosis, a diagnostic algorithm is required. This requires knowledge of a Bayesian method of making a diagnosis. Evidence for usefulness of a diagnostic test must be found. A new diagnostic test, a battery of tests or a prediction rule is tested for its rigour by its sensitivity, specificity, positive predictive value, negative predictive value and likelihood ratios [17-21]. This requires a certain effort. Also, one needs to match it with the availability of the particular diagnostic test at its place of use. Certain tests are operator dependent and would require standardised training to reach the desired level of accuracy. The same test performed with simple CT scan may not compare in terms of diagnosis of hollow abdominal viscera pathology when compared with higher-end-machine with more peripheral attachments like 128 slice tri-phasic triple contrast $\mathrm{CT}$ scan. There is a constant upgradation and development in technology, and the comparative evaluation research cannot keep the pace. 
Similarly, there is also a rapid development in new and modified drugs and pharmaceuticals. For example, to prove superiority of one type of ACE inhibitor or a sartan or a lipidlowering agent with a new salt in the same group after phase I, II and III trials, it is still not known that in a phase IV trial and on a cost-based comparison, both the agents will find their place. Unfortunately a physician who does not understand these finer nuances of EBM can be allured or misguided in prescribing a drug not considering every aspect of the evidence. The pharmaceuticals are able to get into the market and sell a fancy new salt that is slightly different from the established drugs with frills like liposomal, nano, biosimilars and spatial isomers, just by showing fringe benefits at an extra sum. More so, in the use of bio-mimetics like bone cement and prosthetic materials like intra-ocular lens, artificial joints, and coronary stents, much larger sums of money are charged without level I evidence. Such decisions are taken by patients and family on emotional and affordability grounds and the least on available evidence.

A large number of decisions are political. Physicians also in a subtle manner become suggestive inadvertently or for a vested interest. For example, with an extremely low incidence of breast cancer in India ranging from 20 to 60 per one lac adult women population per year as opposed to $200-300$ in the western world, a political decision is likely to be taken to do mass upfront mammographic screening of breast cancer.

Several RCTs that have been ranked in evaluating bi-planer mammography in countries like the UK, the USA and Canada have failed to show great benefit in reducing breast cancer mortality on early detection alone. It is good although such effort can epidemiologically downstage the entire presentation of breast cancer patients. In these countries, more than $90 \%$ subjects present with T1 T2 disease now. In India, majority of patients continue to present with locally advance breast cancer.
An immediate conclusion would be to adopt upfront mammography that has been proposed by many. However, alternatives like breast self-examination and physical examination of the breast by a trained community nurse that was recently tried in Mumbai may be a better strategy. This demonstrates that although there is evidence that use of mammographic screening in western countries results in early detection, it is not applicable to India from a cost-effective point of view for a nationwide breast cancer screening. The improved survival of women with breast cancer is not alone due to early detection and treatment but mostly on account of new therapeutic modalities (www. thelancet.com/lancetgh, vol 6, Aug 2018). It remains to be tested that starting treatment little later does not preclude long-term survival—called lead time bias.

Another gap is in the ranking of evidence which is fundamental to evidence-based practice (EBP). The hierarchy of evidence helps to take a top-down approach of locating the best evidence in a recent well-conducted systematic review. Most experts agree that the higher up the hierarchy the study design is positioned, the more rigorous the methodology and hence the more likely it is that the study design can reduce the effect of bias on the results of the study. In most evidence hierarchies, current, well-designed systematic reviews and meta-analyses are at the top of the pyramid, and expert opinion and anecdotal experience are at the bottom. It is known all too well that landmark developments in surgical techniques, for example, type of prostatectomy, use of laparoscope and more recently increasing use of robotic surgery at a very high cost, are developments that are not from top to bottom but from bottom to top. Only after people will have accumulated series of these surgical operations may one think of doing RCTs.

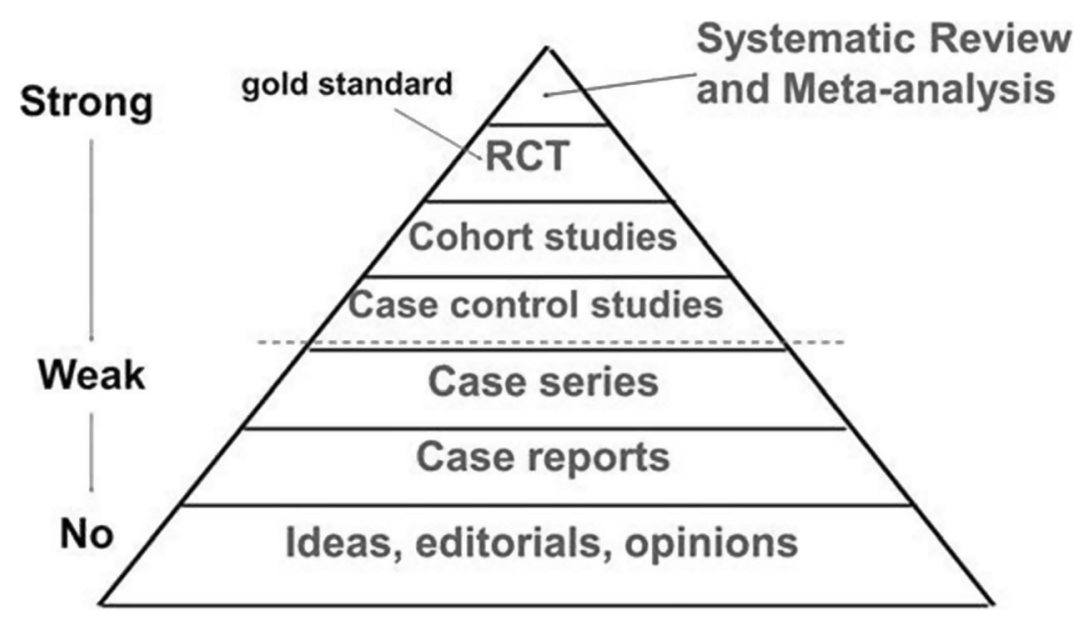




\section{Bayesian Approach to Decision-Making}

Bayesian inference is an important technique in statistics and especially in mathematical statistics. Bayesian inference is a method of statistical inference in which Bayes' theorem is used to update (upgrade or downgrade) the probability for a hypothesis as more evidence or information becomes available [12].

One important task of surgeons is to reach a final diagnosis after doing certain investigations. The investigations are done as parallel tests for routine check-up and comorbidity and initial diagnostic tests including prognostic tests. After analysing all the available evidence from demographic data, clinical data, office examinations and laboratory tests, the next step is planned once you assign in your mind on how much probability for a particular diagnosis am I playing at the moment. The next serially ordered diagnostic test should substantially improve the probability of the diagnosis. Or it should refute/rule out the diagnosis. One now has a post-test diagnostic probability to take a decision for starting the treatment.

Thus, the analysis on known data and prior belief yields a credible value near the sample mean. Further effort in this direction is seeking explicit quantitative use of external evidence to improve the level of certainty. This Bayesian approach is used in design, monitoring, analysis, interpretation and research. This should improve the process of decisionmaking. It also allows a cost effective and a shortest possible route to achieve the highest level of probability.

In research, 3 parameters are required, viz (1) prior distribution which is the probability of a parameter based on previous experience and available trial data, (2) likelihood which is the probability of a parameter based on data from a current ongoing research study or trial and (3) posterior distribution which is the updated probability of the parameter based on our observation in the above method.

\section{Placebo Action in Psycho-Somatic Condition Situation}

A placebo is anything that seems to be an 'actual/real' medical treatment-but is not. It does not contain active ingredients that can affect health or the particular illness. It could be a pill, a shot or some other types of fake treatment or a camouflage. Sometimes placebo can have a response to certain conditions sometimes not, when it combines with counselling and psychosocial belief it works in nearly halves. Up to $60-90 \%$ placebo responses have been reported [22]. A common reason is when patients do embark to take treatment for a condition that they have been neglecting for some time. They also adopt a state of mind to heal themselves, get rid of the problem and institute the life style change that they have been postponing. A common example of this is acute prolapsing haemorrhoids and anal fissures. In the fury of work men continue to slog, do arduous work and eat junk food. When they take large number of prevalent treatments they also take on to a more sagacious routine lifestyle and diet, become more receptive to such advices by their doctor and family. Perhaps the reassurance received from first consultation itself has its healing effect. The specific medical treatment received may actually have no effect on the disease. It is this strategy that is used by commonly advertised treatments in news and social media for conditions like piles, rheumatism, constipation and loss of vigour.

Your mind can be a powerful healer when given the chance. The idea that your brain can convince your body a fake treatment is the real thing - the so-called placebo effectand thus stimulating healing has been around for millennia. Now science has found that under the correct circumstances, a placebo can be just as effective as traditional treatments. The placebo effect is more than positive thinking believing a treatment will work. It is about creating a stronger connection between the brain and body and how they work together. Placebos do not lower cholesterol levels or shrink a tumour. Instead, they work on symptoms modulated by the brain, like the perception of pain. The faith in placebo builds up: 'Placebos may help you feel better, but they will not cure you'. They have been shown to be most effective for conditions like pain management, depression, stress-related insomnia, irritable bowel syndrome and cancer treatment side effects like fatigue and nausea. The placebo is also an add-on effect which enhances the action of an active ingredient used against the disease. For example, in our mastalgia, clinic careful assessment of mastalgia, counselling, eminently ruling out of cancer in breast and high-quality reassurance alone suffice the entire process in majority subjects. In those with residual pain interfering with daily chores of life, effective treatment with active agents like tamoxifen or ormeloxifene work far better and achieve high-quality outcome if the patient of mastalgia also had a highly satisfying and reassuring clinic visit experience [23-25].

\section{Placebo Surgery: More Effective Than You Think?}

Got joint pain? Sham medical procedure is exceptionally viable. Back ache? Sham medical procedure works. Torn meniscus? Have a sham. A trial published in NEJM demonstrated that the phony medical procedure can be in the same class as the genuine $[26,27]$. The subjects were candidates for knee surgery, with a torn meniscus and debilitating pain. Study surgeons in Finland performed either a meticulous repair of the torn cartilage or incisions with no other intervention. In the case anaesthetised patients could hear or understand, the doctors and nurses passed instruments, made surgical sounds and pretended to do surgery for as long as the procedure would normally take. Both surgeries worked at least in the early postoperative period [28].

The effectiveness of fake procedures is a testament to the power of mind and body and a critical window into human healing. But are they practical, or even ethical? The sham 
procedures work in an entirely different spectrum of the same disease - when disease is at a lower level of scale or when it is a chronic condition. Fake procedures work fantastically well in people who have a higher functional overlay and consider allopathic medicine toxic and harmful. Some people scared of surgery may feign benefit after a fake treatment. When someone is having internal bleeding after a car accident, should they be denied a real operation?

Before you reply, consider that surgeons work reflexively, and critics found that many patients bleeding from injury passage best when left to mend without a surgical procedure. A few deaths particularly in children after routine tonsillectomy remind us that surgical procedure should not be messed with. Also, the disclosure that another common medical/surgical procedure is no superior to a sham procedure ought to be a clarion call [29].

\section{Live Fish Medicine in India Claims to Cure Breathing Disorder?}

The Bathini Goud family in Hyderabad hosts a peculiar event every year, where they claim to provide asthamatics with free live fish medicine [30]. Since 175 years, thousands of people from all over the country have been visiting BathiniMrigasira Karthi Fish Prasadam, which takes place on the nights of Mrigasira Karti which falls in June with the onset of the annual monsoon. The $3-\mathrm{cm}$ fish is dipped into a yellow herbal paste and then pressed down the throat of the suffering patients by the members of the Goud family. They claim that the small living fish travels wiggling its way through the human throat, pushing the phlegm and making it easier for people to breathe. Also, the herbal dip used for the asthma medicine remains a secret and is said to be transferred into the Goud Family by a saint in 1845 . About 3 lacs people transcend to the village on a particular moon lit night for fishprasad. Rights activists claim this to be an unhealthy and a ridiculous customary and unlawful event. No attempts have been made to find if there are any medicinal value in the fish and the herbal dip. The ICMR was said to have denied the permission to conduct a trial with the control group of asthma patients who do not receive the fish-prasad taking the frequency of acute asthmatic attacks and drug requirement as outcomes. The argument used was that bronchial asthma is a psycho-somatic disorder and if there is a perceived benefit by a large number of people who continue to go to Goud's fish-prasad, what business do the scientists have to annihilate such a profound faith the practice of which has continued for 175 years.

\section{Clinical Trials-Reliability and Biases [5]}

To what extent may a given treatment work and influence our health? Whenever we decide to take a treatment we want to know how effective it is. Randomised controlled trials are commonly conducted by randomly distributing people into treatment and control groups to test if a treatment may be effective by reducing the bias. Clinical trial is the sanctum sanctorum and hallmark of clinical decision taking. Those who do clinical trials are considered upscale intelligentsia and hard working professionals to improve treatment outcomes. RCT undoubtedly taught us a tidy method of scientific enquiry to settle issues of superiority on equivalence between two drugs. If the sample size or power of study was small we settle for combining the RCTs into meta-analyses. However, in a study conducted at London School of Economics where 10 most influential RCTs worldwide produced biased results [5]. Trials involve complex processes like randomizing, blinding, controlling, monitoring and follow-up. The worst part emerged as the results of trial were carefully watched by the pharmaceutical industry. Market forces led to possible corruption in the trials [4]. In the quest to achieve publishable standards in methodology of RCT like randomisation and blinding, the inclusion criteria get limited and selection bias creeps in further reducing external validity of the results.

Bias is becoming increasingly recognised as a serious problem in many areas of scientific research. Of particular concern are cases in which research results seem directly to reflect the preferences and interests of certain actors/companies involved in the research projects. Troubling examples of this have been identified, especially in privately funded research and in policy-related areas. While some have sought to distinguish acceptable from unacceptable influences of values on science, efforts to draw this distinction in a principled way have proven immensely difficult.

Another bias emerged as 'big fish eating the smaller ones'. An alleged example is that of an efficacious inexpensive selective oestrogen response-modifying agent (SERM) with long half-life [31] — ormeloxifene or centchroman. This was discovered in India in the 1970s. It was tried in the USA when big players propagating SERMS like tamoxifen, anastrozole, letrozole and raloxifene were there. The latter were products of large multi-national pharmaceuticals and were selling at a 10-40 times higher price. A rich dad-poor dad effect forged an ormeloxifene trial to be suspended on a trivial ground.

\section{'Big Data' and 'Real-World Data'}

Collection of extremely large data and information using specialised computational tools and enabling meaningful analysis is the new modality. Data derived from sources like electronic health records, registries, hospital records, health insurance, telephone calls and social media are being said to be both overtly and allegedly secretly subjected to analysis. Such data is used for marketing consumer goods. If health product is a consumer good future decision-making may be influenced by big real world data [32]. 


\section{'The Discordant Note' Trustworthiness of Clinical Research and Randomised Trials}

Dr. Marcia Angel of Harvard Medical School is also the former editor-in-chief of the new England Journal of Medicine has written a book: The Truth About the Drug Companies: How They Deceive Us and What to Do About it. She writes in her book that, "it is no longer possible to believe much of the clinical research that is published, or to rely on the judgement of trusted physicians or authoritative medical guidelines. I take no pleasure in this conclusion, which I reached slowly and reluctantly over my two decades as an editor of The New England Journal of Surgery'. She admitted to systemic corruption where an eminent psychiatrist at Harvard Medical School received millions of dollars for consulting from drug companies to propagate a medicine for frequently over diagnosed bi-polar disorder in children. There was an enormity of conflicts of interests. The three Harvard psychiatrists who were the top in the country and had published research in some of the most important journals have since admitted to accepting millions of dollars from drug companies [4]. Dr. Relman another former Editor-in-chief of NEJM said in 2002 'The medical profession is being bought by the pharmaceutical industry, not only in terms of practice of medicine, but also in terms of teaching and research. The academic institutions of this country are allowing themselves to be the paid agents of the drug companies. I think it is disgraceful'.

Negative trials that do not show benefit of a drug are likely to be suppressed. Favourable results in 36/37 studies were published but only $3 / 36$ with not favourable results were published - a clear example of publication bias. According to one estimate approximately $90 \%$ studies published show favour to use a drug in truth only $50 \%$ actually have a positive report. This is similar to the tendency shown by stock brokers telling you all his winning trades and hiding his loosing trades. So, how much do we trust EBM? Furthermore, in trials done on the behest of the drug companies that do not show favourable results the company quietly chooses not to publish such a study. The journals and the editors are also a part of this conflict. The NEJM editor published a drug funded review article showing use of bis-phosphonates in prevention of femur fracture that had a fine print saying that the study was under powered for definitive conclusions. Some authors of this article were full time employees of the drug company manufacturing bis-phosphonates. Reprints of such articles are purchased by the drug company, advertisements, direct payments from industry and bribery of journal editors have been reported. Besides, publication and selection biases are many ways in which a strong case has been built against some of the most prestigious medical journals. The question now is, do the editors of the NEJM and Lancet believe EBM anymore? The evidence has to be cleaned up from the corrupting influence of industry [33].

\section{Is There a 'Silver Lining' in the Cloud of 'EBM'?}

The discordance in relation to the performance of EBM is not an 'in principle' argument, but it is by chance. If we had unbiased randomised trials we would have better evidence base that, if executed, ought to lead to substantial and measurable health benefits. Although this is a matter of conjecture, it is a hypothesis worth exploring. Looking towards a solution there are plenty of condemnation on the evils of 'Big Pharma', and calls for greater industry accountability and regulation. These approaches are important but it would be naive that these alone can prevent vested interests of the industry. In sport we rely on umpires, not players, to call the penalties. We need to recognise this inherent bias in EBM and need to understand this weakness of EBM. It is beyond the scope of this chapter to discuss practical solution how to overcome bias and industry funded studies in more details.

\section{Quality Improvement}

An emerging new science to improve outcomes and have a win-win situation is the science of translational research and QI (quality improvement) [34]. QI research usually does not have an industry, editor and publisher interface. These directly focus on delivery line. Large multi-centric randomised trials cost millions of dollars and mostly result in a small point estimate of relative benefit. On-site QI exercises efficiently done by honest clinicians and hospital managers can result into huge gain in the outcome. From all the available evidence a judicious pick from clinical research complimented with QI research is the silver lining on the cloud of EBM.

If asked how to improve overall outcome which is the fundamental of healthcare, the suggested responses include the evidence-based medicine (EBM) and clinical quality improvement. EBM has centered more on 'doing the right things' (based on external research evidence) whereas quality improvement (QI) has focused more on 'doing things right' (based on local processes). However, these are complementary and together direct us how to 'do the right things right'. We should 'do the right thing, choose the right modality and do it in the right manner and at the right time', and this age old adage includes both the EBM and QI. QI is a form of clinical process and managerial innovation where local data is used to bring about immediate positive change in the unit or the ward of the hospital. It combines scientific knowledge and applies it to the system, bridging the gap between science and practice. In short it is science of making it happen so that quality care reaches the bedside of the patient.

\section{Evidence-Based Medicine-Where Are We Today?}

Evidence-based medicine as of today is a vexed issue. What was considered judicious and conscientious use of current best evidence to treat individual patients has 
boomeranged and is being questioned by those who regimented it. Every medical practitioner acquires experience and certain clinical expertise. Judicious use of expertise is shared by others who may have somewhat variable experiences and different results. This may require certain adjustments as to when and how to use the experience or expertise of an individual doctor (internal consistency). If this experience is taken up for external testing through research on much larger number of patients and administered by different care providers the external validity of the particular clinical experience/expertise is tested for greater benefit of patients. This external clinical evidence is clinically relevant research. Those who conducted such relevant research and published their data gained respect, became eligible for academic jobs and espoused the practice of EBM. In the process some got corrupted. Research that was laboratory based work is basic science of medicine. These led to new drug development, discovery of biochemical/molecular/genetic basis of disease, evolution of new biomarkers/diagnostic tests/prognostic indicators, etc. Many of these were rewarded with Nobel prize. Clinical research conducted on patients and population allowed computation of efficacy and effectiveness of diagnostic tests, prognostic markers, therapeutic interventions, preventive and rehabilitation regimens.

Rope walking is a technique shown by some with great efficiency. This trick has been in vogue since time immemorial. Similarly, much later someone showed for the first time paddling and balancing on a bicycle as it was invented. Rope walking could not be taken as a means of mass transport because its effectiveness was poor. Whereas a much later discovery of balancing and riding a bicycle is in ubiquitous use ever since. Notably, that there was no trial of efficacy in which a rope walker would have perhaps won and someone might have installed hanging ropes as means of transport and would have utterly failed. Epidemiologists call this efficacy versus effectiveness. All that is efficacious may not be used as an effective tool by people. The contrary is not untrue especially in the world of medicine. Large number of medications are tried even used regularly both by patients, prescribed by doctors which are neither efficacious nor have been used effectively and may have failed at the altar of randomised trials. As David Sackett in 1996 said evidence-based medicine is not a 'cook book' medicine. It requires a bottom-up approach that integrates the best external evidence with individual expertise, available resources and patients' choice [1]. It does not enslave the clinician. External clinical evidence especially from randomised trials can inform, but can never replace, individual clinical wisdom and clinical decision taken in right earnest. All-out effort to deliver healthcare with confidence and smile requires quality improvement exercises and an honest effort over all.

\section{References}

1. Sackett DL, Rosenberg WM, Gray JA, Haynes RB, Richardson WS (1996) Evidence based medicine: what it is and what it isn't. BMJ 312:71-72

2. Guyatt GH (1991) Evidence-based medicine. ACP J Club 114:A-16

3. Szajewska H (2018) Evidence-based medicine and clinical research: both are needed, neither is perfect. Ann Nutr Metab 72(suppl 3): 13-23

4. Thomas Caroline November 9, 2002. NEJM editor: no longer possible to believe much of clinical research published. https:// ethicalnag.org/2009/11/09/nejm-editor/

5. Krauss A (2018) Why all randomised controlled trials produce biased results. Ann of Med 50(4):312-322

6. McCulloch P, Taylor I, Sasako M (2002) Randomised trials in surgery: problems and possible solutions. BMJ 324:1448-1451

7. Rastogi S. Comparative effectiveness research: appropriating the benefits of traditional health care referring Ayurveda. Comparative Effective Research 2016; Chapter 21 : 507-516. ISBN 978-1-63484-315-7

8. Mirghafourvand M, Alizadeh MCS, Ahmadpour P, Javadzadeh Y (2016 Feb) Effect of Vitex agnus and flaxseed on cyclic mastalgia: a randomized controlled trial. Complement Therap Med 24:90-95. https://doi.org/10.1016/j.ctim.2015.12.009

9. Oxford Centre for Evidence-based Medicine (2001) Levels of evidence. Available at: http://www.cebm.net/index.aspx? O = 1025Cochrane AL (1972) Effectiveness and efficiency: random reflections on health services. Nuffield Provincial Hospitals Trust, London

10. Lubin JH, Adams MJ, Shore R, Holmberg E, Schneider AB, Hawkins MM, Robison LL, Inskip PD, Lundell M, Johansson R, Kleinerman RA, de Vathaire F, Damber L, Sadetzki S, Tucker M, Sakata R, Veiga LHS (2017) Thyroid cancer following childhood low-dose radiation exposure: a pooled analysis of nine cohorts. J Clin Endocrinol Metab 102:2575-2583

11. Practicing EBM - the 5-step approach. https://canberra.libguides. com/evidence

12. Petrisor BA, Bhandari M (2007) The hierarchy of evidence: levels and grades of recommendation. Indian J Orthop 41(1):11-15

13. Ashrafian H, Sevdalis N, Athanasiou T (2010) Evidence based surgery. Key topics in surgical research and methodology. Springer-Verlag, Berlin Heidelberg. https://doi.org/10.1007/978-3540-71915-1 2

14. National Health and Medical Research Council (1999) A guide to the development, evaluation and implementation of clinical practice guidelines. Available at: http://www.nhmrc. gov.au/publications/ synopses/_files/cp30.pdf

15. Marshall JC (2006) Surgical decision-making: integrating evidence, inference, and experience. Surg Clin North Am 86:201-215 xii

16. Garrett M (2017) Bassini repair of inguinal hernia. In: Hoballah J, Scott-Conner C, Chong H (eds) Operative dictations in general and vascular surgery. Springer, Cham

17. Kumar R, Misra PK, Kumar S (1992) Testing a test. Ind Pediatrics 29:667-672

18. Kumar R, Kumar S (1993) Research strategies for the clinician. Ind Pediatrics 30:1041-1048

19. Kumar R, Kumar S (2004) Evaluation of diagnostic tests in breast cancer. Mammology 1(1):17-19

20. Rai R, Dwivedi V, Kumar R, Kumar S (2005) Questionnaire Designing. Mammology 1(2):22-26

21. Kumar S, Arun D (2000) Surgical audit (editorial). Nat Med J Ind 13:113-114

22. Paul E, Bjoern H, Weimer K, Klosterhalfen S (2012) Placebo responses and placebo effects in functional bowel disorders. Eur $\mathbf{J}$ Gastroenterol Hepatol 24(1):1-8 
23. Kumar S, Rai R, Agarwal GG, Dwivedi V, Kumar S, Das V (2013) A randomised double blind placebo controlled clinical trial of Centchroman (Ormeloxifene) in breast pain and nodularity (benign breast disorder). Natl Med J India 26:69-74

24. Kataria K, Dhar A, Srivastava A, Kumar S, Goyal A (2014) A systematic review of current understanding and management of Mastalgia. Indian J Surg 76(3):217-227. https://doi.org/10.1007/ s12262-013-0813-8

25. Wisbey JR, Kumar S, Mansel RE, Preece PE, Pye JK, Hughes LE (1983; ii) Natural history of breast pain. Lancet 322:672-74A

26. Kirkley A, Birmingham TB, Litchfield RB, Giffin JR, Willits KR, Wong CJ, Feagan BG, Donner A, Griffin SH, D'Ascanio LM, Pope JE, Fowler PJ (2008) A randomized, controlled trial of arthroscopic surgery for osteoarthritis of the knee. N Engl J Med 359:1097-1107

27. Moseley JB, O'Malley K, Petersen NJ et al (2002) A controlled trial of arthroscopic surgery for osteoarthritis of the knee. N Engl J Med 347:81-88

28. Marx RG (2008) Arthroscopic surgery for osteoarthritis of the knee? N Engl J Med 359:1169-1170

29. Windfuhr JP, Schloendorff G, Baburi D, Kremer B (2008) Lethal outcome of post-tonsillectomy hemorrhage. Eur Arch Otorhinolaryngol 265:1527-1534
30. Murthy PSR and Priyadarsini K, Jyothsna, A Voyage of medical tourism through alternative medicine (with reference to Bathini fish medicine in Hyderabad) (January 12, 2011). Available at SSRN: https://ssrn.com/abstract=1738802 or https://doi.org/10.2139/ssrn. 1738802

31. Gara RK, Sundram V, Chauhan SC, Jaggi M (2013) Anti-cancer potential of a novel SERM Ormeloxifene. Curr Med Chem 20(33): 4177-4184

32. Ward JS, Barker A. Undefined by data: a surgery of big data definitions. http://arxiv.org/abs/1309.5821

33. Fung Jason (2018) The corruption of evidence based medicine killing for profit 2018. https://medium.com/@drjasonfung/thecorruption-of-evidence-based-medicine-killing-for-profit41f2812b8704 (accessed October 20, 2018)

34. Abdelwahab R, Yang H, Teka HG (2017) A quality improvement study of the emergency center triage in a tertiary teaching hospital in northern Ethiopia. African J Emerg Med 7:160-166

Publisher's Note Springer Nature remains neutral with regard to jurisdictional claims in published maps and institutional affiliations. 